\title{
Compliance evaluation of removable space maintainer or space regainer usage
}

\author{
Revanti Ramadhani*, Syarief Hidayat*, Risti Saptarini Primarti* \\ *Department of Pedodontics Faculty of Dentistry Universitas Padjadjaran, Indonesia
}

\begin{abstract}
Premature loss could cause a problem with the tooth arrangement or the dental arch size. A space left by the primary tooth loss could cause migration of the adjacent teeth. As a result, space will be narrowed and undermined the eruption of the permanent teeth. The success of the space maintainer or space regainer usage due to the premature loss marked by space for the replacement of the permanent teeth. The purpose of this research was to evaluate the compliance of children in wearing a space maintainer or space regainer after insertion at Pedodontics Installation of Faculty of Dentistry Universitas Padjadjaran Dental Hospital, Bandung, Indonesia. The research method was descriptive survey technique. The sample consisted of 30 patients selected using the total sampling technique. Data were obtained with a questionnaire and statistically analyzed. The results showed that majority of the children uses the removable space maintainer or the space regainer daily was only about $23,3 \%$ overall. Most of the children only use the removable space maintainer or the space regainer for sometimes. The research concluded that the low rate of pedodontic patients compliance at Pedodontics Installation of Faculty of Dentistry Universitas Padjadjaran Dental Hospital in the usage of the removable space maintainer or the space regainer was usually caused by pain or discomfort. This fact was evidence of a low awareness of parents in preventing malocclusion to their children.
\end{abstract}

Keywords: Premature loss, patient compliance evaluation, space maintainer, space regainer

\section{INTRODUCTION}

Premature loss could cause a problem with the tooth arrangement or the dental arch size. A space left by the primary tooth loss could cause migration of the adjacent teeth. As a result, space will be narrowed and undermined the eruption of the permanent teeth. Permanent teeth will erupt out of the dental arch, resulting in crowded teeth or malocclusion. For example, the premature loss of primary molar usually followed by the migration of the first permanent molar to the mesial, which was observed six months after the tooth extraction. ${ }^{1}$

Premature loss caused side effect towards the jaws growth and development. Prevention and reduction of the premature loss should be done to prevent malocclusion. The use of the space maintainer and space regainer can be done or the treatment of premature loss. A space maintainer is useful to keep the space due to the tooth loss to prevent the adjacent tooth migration. Space maintainer can effectively work to prevent malocclusion because of space 
reduction due to premature loss of the primary tooth. ${ }^{2}$ The right time to use the space maintainer or space regainer is immediately after the loss of a primary tooth because there will be a closure of the space six months after the tooth loss.

There are several types of space maintainer, generally divided into two categories, removable and fixed. ${ }^{3}$ Removable space maintainer was used when the tooth loss occurred in more than one tooth in a quadrant. ${ }^{1}$ This type of space maintainer can be used and removed by the patients themselves but has some weaknesses such as easily broken and lost, thus made the device becomes ineffective to keep the space. ${ }^{4}$ The most commonly used removable space maintainer is the Hawley Plates/Hawley Retainer. The fixed space maintainer is the space maintainer that installed firmly in the patient's mouth and could only be removed by dentists. ${ }^{5}$ The most commonly used fixed space maintainers are band and loop, crown and loop, lingual arch and distal shoe. ${ }^{1}$

The space regainer is an interceptive orthodontic appliance used to widen the narrowed space by pushing the tooth back to the normal position, resulting in enough space for the permanent tooth eruption. This kind of treatment could prevent any crowded teeth or tooth impaction.6 This treatment usually performed in patients with the mixed dentition stage, which vulnerable most towards the risk of malocclusion.

The success of the space maintainer or space regainer will be achieved when the children continue to use it until the desired space obtained, and the permanent tooth erupted at place. Children often feel lazy to use the devices because it felt less comfortable, and also because of the parents less awareness regarding of its importance. Tulunoglu et al. had conducted a retrospective study for six years on 633 patients aged 4-15 years old who used several types of space maintainer. This study showed that as much as $52.7 \%$ of the patients did not continue the treatment, $12.7 \%$ failed on treatment, $3 \%$ still using the device when the study conducted, and only as much as $31.5 \%$ of the patients were successfully finished the treatment and no longer needed the device. This study also discovered that the fixed space maintainer metal attachment was broken and the loop tip was pressing the gum tight enough resulted in gingival inflammation.
The most common failures in removable space maintainer treatment were usually a deformation in the retentive device resulting in reduced retention.

In Pedodontics Installation of Faculty of Dentistry Universitas Padjadjaran Dental Hospital, Bandung, Indonesia, space maintainer or space regainer application is one of the clinical requirements of undergraduate dentistry students. The students have to do twice insertion and controlling the space maintainer or space regainer usage after insertion until the eruption of the permanent teeth. The usage evaluation, however, had not been performed before. The purpose of this research was to evaluate the compliance of children in wearing a space maintainer or space regainer after insertion at Pedodontics Installation of Faculty of Dentistry Universitas Padjadjaran Dental Hospital, to obtained data for clinical evaluation and recommendation of improvement in undergraduate dentistry education program.

\section{METHODS}

The research method was a descriptive survey technique. The sample consisted of 30 patients selected using the total sampling technique. Data obtained by questionnaires regarding the information of the space maintainer or space regainer usage post insertion at Pedodontics Installation of Faculty of Dentistry Universitas Padjadjaran Dental Hospital in August 2011. The population of this research pedodontic patients at Pedodontics Installation of Faculty of Dentistry Universitas Padjadjaran Dental Hospital using a space maintainer or space regainer. Materials and tools used for this study were stationery, informed consent, and questionnaires.

The research variable was the compliance evaluation of the space maintainer or space regainer usage in pedodontic patients post insertion. The operational definition of the space maintainer was a device used to maintain the space occurred due to the premature loss of the primary tooth, whereas the space regainer was a device used to realign the tooth position that already moved to the mesial part (mesial drifting) to make the permanent tooth erupted at place. Insertion was the stage of installation of the device into the oral cavity which was performed after polishing the 
device. The compliance evaluation of the space maintainer or space regainer usage was done by giving questionnaires to be filled by the patients, then the data obtained were recorded, compiled, processed, and presented in tables and figures.

\section{RESULTS}

The respondent's characteristic in this study included gender and age of the patients presented in Figure 1.
Figure 1 above showed that most pedodontic patients were male. There were found only as much as 11 female patients (36.7\%).

Respondents distribution by age presented in Figure 2, showed that the most age group of pedodontic patients using a space maintainer or space regainer at Pedodontics Installation of Faculty of Dentistry Universitas Padjadjaran Dental Hospital were the 8-years-old age group, which was as much as 11 patients (36.7\%) from total sample. The fewest age group was the 6-years-

Table 1. Space maintainer or space regainer usage

\begin{tabular}{|c|c|c|}
\hline The use of space maintainer/regainer & Frequency & Percentage \\
\hline \multicolumn{3}{|l|}{ Using space maintainer/regainer every day: } \\
\hline Yes & 7 & $23.3 \%$ \\
\hline No & 23 & $76.7 \%$ \\
\hline Total & 30 & $100 \%$ \\
\hline \multicolumn{3}{|c|}{ If not, what time you use space maintainer/regainer: } \\
\hline Often (5-6 days in a week) & 2 & $8.7 \%$ \\
\hline Occasionally (3-4 days in a week) & 12 & $52.2 \%$ \\
\hline Rarely ( $1-2$ days in a week) & 5 & $21.7 \%$ \\
\hline Never & 4 & $17.4 \%$ \\
\hline Total & 23 & $100 \%$ \\
\hline \multicolumn{3}{|c|}{ Reasons not to use space maintainer/regainer every day: } \\
\hline Space maintainer/regainer was missing & 0 & 0 \\
\hline Not reminded by operator & 2 & $9.1 \%$ \\
\hline Lazy & 4 & $18.2 \%$ \\
\hline Pain / discomfort & 14 & $63.6 \%$ \\
\hline Tooth already erupted / loss of adjacent tooth & 2 & $9.1 \%$ \\
\hline Total & 22 & $100 \%$ \\
\hline \multicolumn{3}{|l|}{ Know the function of space maintainer/regainer: } \\
\hline Yes & 16 & $53.3 \%$ \\
\hline No & 14 & $46.7 \%$ \\
\hline Total & 30 & $100 \%$ \\
\hline \multicolumn{3}{|c|}{ If so, what is the function of space maintainer/regainer: } \\
\hline Keep spaces between teeth & 7 & $43.8 \%$ \\
\hline Create spaces between teeth & 3 & $18.8 \%$ \\
\hline Fix the tooth position & 5 & $31.3 \%$ \\
\hline Moving the teeth & 1 & $6.3 \%$ \\
\hline Total & 16 & $100 \%$ \\
\hline \multicolumn{3}{|c|}{ Did operator told the function of space maintainer/regainer: } \\
\hline Yes & 17 & $56.7 \%$ \\
\hline No & 13 & $43.3 \%$ \\
\hline Total & 30 & $100 \%$ \\
\hline \multicolumn{3}{|c|}{ Did your parents told you to always use the space maintainer/regainer: } \\
\hline Yes & 9 & $30.0 \%$ \\
\hline No & 21 & $70.0 \%$ \\
\hline Total & 30 & $100 \%$ \\
\hline
\end{tabular}




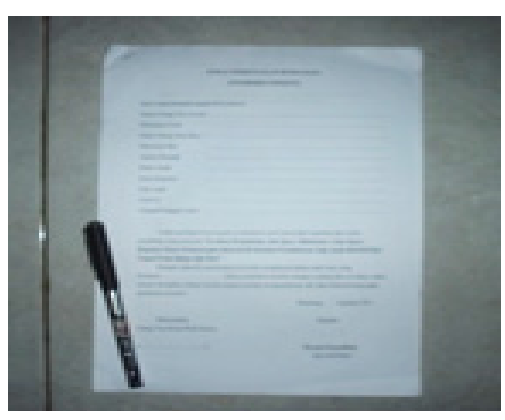

Figure 1. Research materials and tools

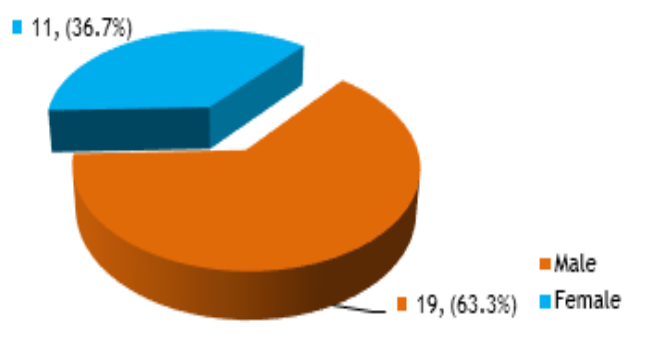

Figure 1. Respondents distribution by gender

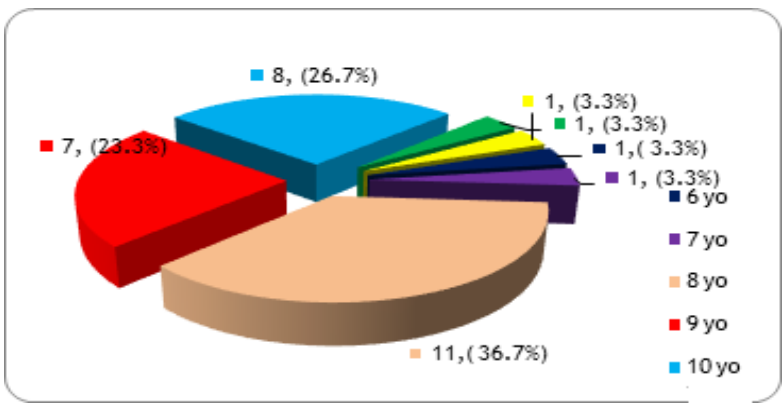

Figure 2. Respondents distribution by age

old, 7-years-old, 11-years-old, and 12-years-old age groups, which were respectively as much as 1 patient (3.3\%).

Table 1 presented the pedodontics patients compliance evaluation of the space maintainer or space regainer usage at Pedodontics Installation of Faculty of Dentistry Universitas Padjadjaran Dental Hospital.

The results from the pedodontics patients compliance evaluation of the space maintainer or space regainer usage post insertion until the eruption of permanent teeth showed that only a few respondents who are continuously using their space maintainer or space regainer daily (Table 1). Most of the patients were using their space maintainer or space regainer occasionally, ranged from 3-4 days weekly.

The main reason for the children not using the devices daily was due to the pain or discomfort feelings and laziness. The children's lack of knowledge regarding the space maintainer or space regainer function was indicated by the number of children who claimed their ignorance $(46.7 \%)$. Most of the children were considering the space maintainer or space regainer was the device used to maintain spaces between teeth, and the others were considering the function was to fix the tooth position. Almost half of the respondents (43.3\%) admitted that they did not get any information regarding the space maintainer and space regainer function from the operator. Similarly, most of the parents did not tell or suggest their children to keep using the space maintainer or space regainer daily.

\section{DISCUSSION}

This study was evaluating the compliance of pedodontic patient's in the usage of the space maintainer or space regainer post insertion. The tooth eruption in female children was faster than male children thus increased the caries rate in female children. ${ }^{7}$ This theory, however, was not consistent with the results obtained in this study which showed that the space maintainer or space regainer user was found more in male children. Data showed that as much as 19 patients $(63.3 \%)$ from pedodontic patients using a space maintainer or space regainer at Pedodontics Installation of Faculty of Dentistry Universitas Padjadjaran Dental Hospital was male children (Figure 1). This condition could be caused diligency of female children on brushing their teeth and maintaining their healthy dietary habit than male children, which also causing lower caries rates in female children of this research population.

The most frequent age group using a space maintainer or space regainer was the 8-years-old group (Figure 2). According to Schroeder, ${ }^{8}$ in the 8-years-old age group, posterior teeth experienced premature loss will be replaced with the maxillary and mandibular first and second premolar, which will erupted at the age of 9-10 years old. The space maintainer or space regainer usually applied to maintain the space until the permanent tooth erupted. The study conducted by Halimah9 amongst 100 children at Pedodontics Installation of Faculty of Dentistry Universitas Padjadjaran Dental Hospital showed that the premature loss of 
the posterior primary tooth was as much as $44 \%$. Pedodontics Installation of Faculty of Dentistry Universitas Padjadjaran Dental Hospital only treated posterior tooth premature loss by the installation of the removable space maintainer or space regainer.

Based on the questionnaire of this research, concluded that daily usage of the space maintainer or space regainer was very low (23.3\%) (Table $1)$. Most of the patients $(52.2 \%)$ stated that the space maintainer or space regainer only used occasionally. Patients used the device often was as much as $8.7 \%$, rarely used the device $21.7 \%$, and $17.4 \%$ of the patients never used the device (Table $1)$. Good communication was one of the ways to make children use their space maintainer or space regainer daily. ${ }^{10}$ Removable space maintainer or space regainer should be used daily, except when eating or brushing the teeth. The role of parents also very important, thus the operator must gave explanations to the parents to monitor the children's compliance on the treatment.

The main reason for children not using the space maintainer or space regainer daily was pain or discomfort, found in as much as $63.6 \%$ of respondents (Table 1). Unscheduled control of the usage of the space maintainer or space regainer by the operator would cause destruction of the soft tissue if there were any piercing wire or a sharp and compressive part. ${ }^{11}$ As much as $18.2 \%$ of the children were feeling lazy to use the device (Table 1). In the first week of the usage, patients will usually feel discomfort. Therefore, the operator should inform the patients to adapting to the device. ${ }^{4}$ As much as $9.1 \%$ of the children stated that the operator did not give their space maintainer or space regainer, which was very regrettable because the appliance should be given to the patient after the last control visit. As much as $9.1 \%$ of the children stated that their permanent tooth had already erupted thus the space maintainer or space regainer was not needed anymore. There were no children stated that their space maintainer or space regainer was lost (Table 1).

The result of this study was consistent with the study conducted by Rajab1 which evaluated the clinical performance of 387 space maintainer devices on 358 children aged 3-9 years olds in
Pedodontics Installation of Faculty of Dentistry University of Yordania from 1996 until 2000. The failure occurred in 119 appliances (30.7\%), of which $49.6 \%$ were due to solder breakage, $32.8 \%$ from cement loss, $11 \%$ from soft tissue lesions, 4.2\% from eruption interference, and $2.5 \%$ were completely lost. By using the Kaplan-Meier method, the estimated median survival time for space maintainers was 18 months. Lingual arches had the lowest median survival time of 14 months. Bands and loops, Nance appliances, and removable partial dentures had a similar probability of survival. Fixed bilateral mandibular appliances recorded lower survival time than fixed bilateral maxillary appliances. ${ }^{2}$

The children's knowledge about the usage of a space maintainer or space regainer was still low because as much as $46.7 \%$ of the children admitted that they did not know the device's function. This condition could become one of the factors causes the irregular usage of the space maintainer or space regainer. The questionnaire results showed that most of the children (43.3\%) did not know the function of the space maintainer or space regainer because the operator did not explain it (Table 1). The function of the space maintainer is to prevent tooth migration due to the premature loss. ${ }^{6}$ The function of the space regainer was to repositioned the teeth to obtain a space for the permanent tooth eruption. ${ }^{12}$ This theory was consistent with the children's knowledge obtained from this study $(43,8 \%)$ (Table 1). This consideration could be based on the operator's explanation regarding the device's function, thus encouraged the children to not only uses the device but also knows the benefits.

There were only $30 \%$ of the parents who told their children to use the space maintainer or space regainer daily, whilst the rest of the children admitted that their parents never told them to do so (Table 1). The lack knowledge of the parents on the importance of the space maintainer or space regainer usage caused by the operator's negligence on informing the parents regarding the importance of periodic treatment. Operator should give the parents information regarding their children's oral condition and the possibility of malocclusion through pictures, radiographic images, and dental model. ${ }^{13}$ 


\section{CONCLUSION}

Pedodontic patients compliance at Pedodontics Installation of Faculty of Dentistry Universitas Padjadjaran Dental Hospital in the usage of the removable space maintainer or the space regainer was very low. Discomfort or pain was the main reason to not using the space maintainer or space regainer.

\section{REFERENCES}

1. Pinkham JR. Pediatric Dentistry: Infancy Through Adolescence. $4^{\text {th }}$ ed. Philadelphia: Saunders-Elsevier; 2005. p. 423.

2. Rajab LD. Clinical performances and survival of space maintainer evaluation over a period of 5 years. ASDC J Dent Child. 2002 MayAug;69(2):156-60.

3. Foster TD. Buku Ajar Ortodonsi. $3^{\text {rd }}$ ed. Jakarta: EGC; 1997. p. 124.

4. Cameron AC, Widmer RP. Handbook of Pediatric Dentistry. $2^{\text {nd }}$ ed. St. Louis: MosbyElsevier; 2003. p. 293-4, 297.

5. Jablonsky S. Illustrated Dictionary of Dentistry.
Philadelphia: Saunders-Elsevier; 1982.

6. Moyers RE. Handbook of Orthodontics for The Students and General Practitioner. $4^{\text {th }}$ ed. Chicago: Year Book Medical Publishers, Inc.; 1991. p. 159-62.

7. Finn SB. Clinical Pedodontics. $4^{\text {th }}$ ed. Philadelphia: Saunders-Elsevier; 2000. p. 327.

8. Welbury R, Duggal MS, Hosey MT. Paediatric Dentistry. $2^{\text {nd }}$ ed. Oxford: Oxford University Press; 2003.

9. Halimah A. Frekuensi Premature Loss Gigi Sulung pada Anak Usia 4-9 Tahun di Bagian Kedokteran Gigi Anak RSGM FKG UNPAD Februari 2009 [minor thesis]. Bandung: Unpad; 2009.

10. Dean JA, Avery DR, MCDonald RE. Dentistry for The Child and Adolescent. $7^{\text {th }}$ ed. St. Louis: Mosby-Elsevier; 2000.

11. Foster TD. Buku Ajar Ortodonsi. $3^{\text {rd }}$ ed. Jakarta: EGC; 1997. p. 124.

12. Proffit W, Fields H. Contemporary Orthodontics. $4^{\text {th }}$ ed. St. Louis: Mosby-Elsevier; 2007. p. 76, 93-4.

13. Brauer JC. Dentistry for Children. $5^{\text {th }}$ ed. New York: McGraw-Hill; 1964. p. 403. 